\title{
Early implementation of Fundamentals of Endoscopic Surgery training using a simulation-based mastery learning curriculum
}

\author{
Christopher Dyke, MD*, Brenton R. Franklin, MD, MHPE, FACS, \\ W. Brian Sweeney, MD, FACS, E. Matthew Ritter, MD, MHPE, FACS
}

\section{A R T I C L E I N F O}

\section{Article history:}

Accepted 7 December 2020

Available online 11 February 2021

\begin{abstract}
A B S T R A C T
Background: After the American Board of Surgery announcement of the Flexible Endoscopy Curriculum requirement in 2014, we implemented a dedicated endoscopy rotation at the post graduate year (PGY)2 level including a simulation curriculum for Fundamentals of Endoscopic Surgery skills. Here we evaluate the outcomes of this implementation.

Methods: Beginning in 2015, we developed a clinical endoscopy and simulation-based rotation to prepare for Fundamentals of Endoscopic Surgery testing. Originally, our curriculum was based on the published Texas Association of Surgical Skills Laboratories curriculum using the GI Mentor and transitioned to a mastery learning curriculum using the Endoscopy Training System in 2016. We evaluated the success of the curriculum in terms of first-time pass rates, training time required, and comparison to previously published benchmarks based on clinical experience.

Results: Since 2015, a total of 37 general surgery residents in our program were Fundamentals of Endoscopic Surgery tested (PGY2 = 24, PGY3 = 4, PGY5 = 9); 84\% (31) completed the Endoscopy Training System curriculum. At the time of testing, $73 \%$ (27) had performed $<25$ esophagogastroduodenoscopies, and $46 \%$ had performed $<25$ colonoscopies. Ninety-two percent (34) spent 10 hours or less completing the curriculum. The first-time pass rate for those completing the Endoscopy Training System curriculum was $97 \%$ vs $67 \%$ for those not completing the Endoscopy Training System curriculum $(P=.01)$. For residents completing the Endoscopy Training System curriculum, total Fundamentals of Endoscopic Surgery scores were discernibly higher ( 472 vs $389, P<.01$ ), as were $3 / 5$ task scores (Nav1 80 vs $67, P=$ .02 ; Loop2 36 vs $8, P=.02$; Retro3 89 vs $71, P=.02$ ). Despite clinical inexperience ( $<25$ esophagogastroduodenoscopies and $<50$ colonoscopies), PGY2s yielded a mean score of 454 and a pass rate of $92 \%$. This was similar to PGY5s (427, 89\%; $P=.3$ ) and compares to benchmark data of endoscopists with $>300$ cases.

Conclusion: Early implementation of flexible endoscopy training with a simulation-based curriculum results in Fundamentals of Endoscopic Surgery performance equal to a clinical experience not often gained during surgical residency. Often requiring $<10$ hours, this represents a fantastic return on investment for this training.
\end{abstract}

Published by Elsevier Inc.

\section{Introduction}

In 2014 the American Board of Surgery (ABS) announced new requirements for general surgery board eligibility, which included completion of the Flexible Endoscopy Curriculum (FEC). Key FEC components include a dedicated endoscopy rotation, performing at least 35 upper endoscopies and 50 colonoscopies, and obtaining a passing score on the

\footnotetext{
* Reprint requests: Christopher Dyke, MD, Department of General Surgery, Walter Reed National Military Medical Center, 8901 Wisconsin Ave, Bethesda, MD 20889.

E-mail address: dyke.christopher@gmail.com (C. Dyke).
}

Fundamentals of Endoscopic Surgery (FES) exam developed by the Society of American Gastrointestinal and Endoscopic Surgeons (SAGES). ${ }^{1}$ These new requirements applied to general surgery residents graduating in 2018 and after.

The FEC published by the ABS outlines a stepwise, milestonebased curriculum for residents to obtain the knowledge and skills required for safe endoscopic surgery. Strong recommendations were made for the length of the endoscopy rotation to be at least 1 month and for completion of the FEC no later than the end of the fourth post graduate year (PGY) of training. ${ }^{1}$ As a result, curricula using both virtual reality and physical endoscopy training simulators were developed and instituted by general surgery programs across the nation. $^{2-5}$ 


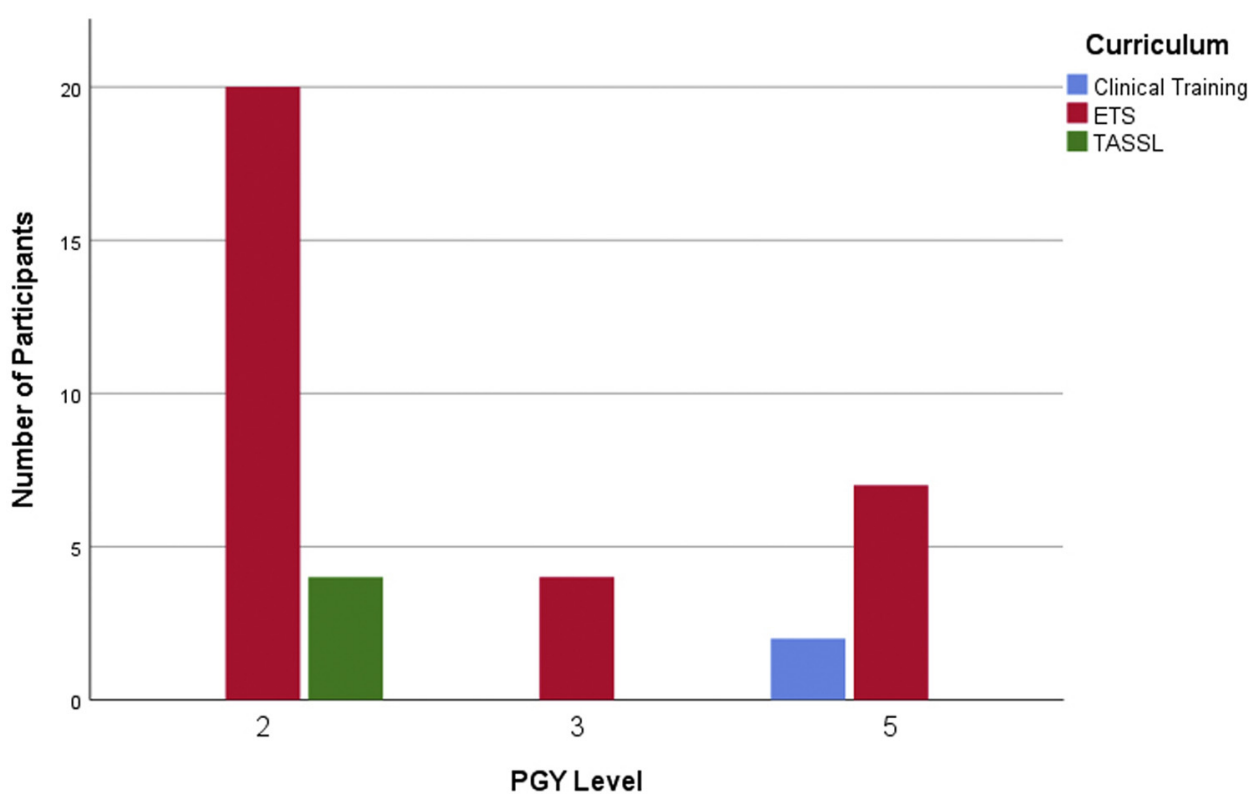

Fig 1. Summary of curriculum used by PGY level.

Even with clinical endoscopy experience combined with institution-specific resident endoscopy training, 1 study showed that $25 \%$ of graduating chief residents failed to achieve a passing score on the skills portion of the FES exam on the first attempt despite meeting the ABS endoscopic case requirement minimums. ${ }^{6}$ It has also been suggested that clinical endoscopy experience of at least 60 colonoscopies and 103 total endoscopies was a predictor of a passing score on the FES skills examination. ${ }^{6}$ Results continue to be inconsistent, and hypotheses remain that low first-time pass rates on the FES examination are due to clinical inexperience.

Beginning in 2015, we implemented a month-long rotation dedicated to surgical simulation and clinical endoscopy (EndoSim, ES) at the PGY2 level. In 2015, endoscopic-specific simulation training during the ES rotation was based on the Texas Association of Surgical Skills Laboratories (TASSL) curriculum and used the virtual reality GI Mentor II (Simbionix USA, Cleveland, OH) endoscopic trainer. ${ }^{8}$ In 2016, our ES rotation adopted a simulation-based mastery learning (SBML) curriculum using the Endoscopy Training System (ETS; Limbs and Things LLC, Bristol, UK). ${ }^{5,9}$ Briefly, this curriculum uses 2 tabletop physical endoscopy simulator platforms and a standard endoscope to train 5 skills: scope manipulation, tool targeting, retroflexion, loop management, and mucosal inspection. Preparation for the cognitive portion of the FES examination included completion of the online FES didactic modules with additional supplemental readings.

Our goal was to assess the outcome of implementation of the ES rotation and the SBML curriculum early in our residency program at the PGY2 level.

\section{Methods}

Deidentified FES skills exam data were obtained with permission from the SAGES FES Program for all test takers registering as Walter Reed general surgery residents who completed FES testing from 2015 to 2019. Before FES testing, general demographic information and self-reported clinical endoscopy experience estimates were obtained. Clinical endoscopic experience for upper (esophagogastroduodenoscopy, percutaneous endoscopic gastrostomy) and lower (colonoscopy, flexible sigmoidoscopy) was stratified into ranges of $0-24,25-49,50-99,100-199$, and greater than 200. Time to completion of the simulation curriculum (where applicable) was self-reported into stratified ranges of $<1,1$ to 5,6 to 10 , 11 to 20 , and $>20$ hours. Additionally, total and task-specific FES scores and first-time pass rates were abstracted and analyzed.

Starting in academic year (AY) 2015, PGY2 residents participate in a dedicated endoscopy rotation, including a simulation-based training curriculum (ES). The rotation includes clinical performance of upper endoscopies and lower endoscopies with surgeon faculty members. The simulation curriculum includes training to prepare for the Fundamentals of Laparoscopic Surgery ${ }^{10,11}$ skills test and a simulation-based robotic surgery curriculum in addition to flexible endoscopy. For the 2015-2016 AY, residents completed the TASSL curriculum on the GI Mentor II (Simbionix USA, Cleveland, $\mathrm{OH}$ ). Beginning in the 2016-2017 AY, residents on the ES rotation transitioned to the ETS curriculum. ${ }^{5}$ Residents who did not have the opportunity to complete the ES rotation (ie, PGY 3 or higher in AY 2015) were provided the opportunity to complete the ETS curriculum before FES testing. As a program requirement, residents were required to successfully complete a simulationbased curriculum to receive an FES test voucher from the program. Residents not wishing to complete either curriculum purchased test vouchers with their personal funds. Residents who competed the ES rotation underwent FES testing as a PGY2. Otherwise, testing was done at the PGY3 level or above based on resident preference.

Statistical analysis was performed using IBM SPSS Statistics, version 25. Means were compared for normally distributed continuous variables using independent samples $t$ tests or 1-way analysis of variance as appropriate. Proportional data were compared using $\chi^{2}$ or Fisher exact test as indicated by subject number.

\section{Results}

Thirty-seven general surgery residents completed the FES exam. Two (5\%) trained with clinical endoscopy only without 

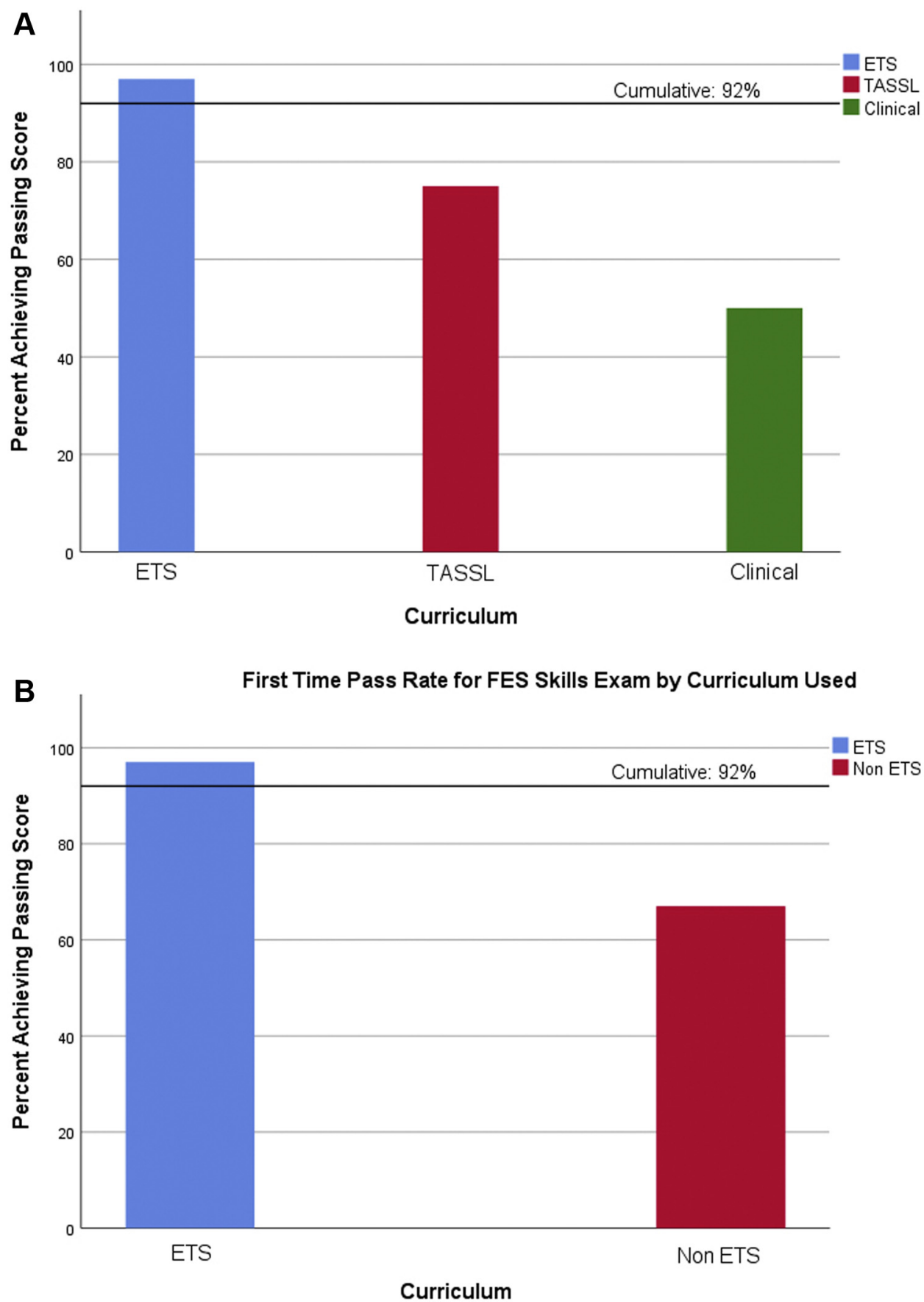

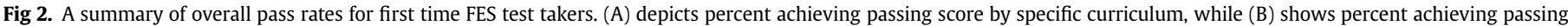
score for the groups divided into ETS and non ETS curricula. FES, Fundamentals of Endoscopic Surgery.

an ES rotation or simulation training, $4(11 \%)$ completed the ES rotation using the TASSL curriculum on the GI Mentor virtual reality simulator, and 31 (84\%) completed the ETS SBML curriculum (Fig 1). At the time of testing, 24 (65\%) were at the PGY2, $4(11 \%)$ at the PGY3, and $9(24 \%)$ at the PGY5 training level. All 24 PGY2 residents (65\%) tested had completed the ES rotation.

At the time of FES testing, 17 (46\%) residents had performed $<25$, 10 (27\%) had performed 25 to 49,9 (24\%) had performed 50 to 99 , and 1 resident reported performing $>100$ lower endoscopies. Similarly, 27 residents (73\%) had performed fewer than 25,8 (22\%) had performed 25 to 49, and 2 (5\%) had performed 50 to 99 upper endoscopies.

The cumulative first-time FES test pass rate for our program was 92\%; however, the first-time pass rate for residents who completed the ETS curriculum was $97 \%$ (30 of 31 ) vs $75 \%$ ( 3 of 4 ) for the TASSL curriculum vs $50 \%$ ( 1 of 2 ) for no simulation curriculum (Fig 2, A). Given the small number of participants in the latter 2 groups, they were combined into a non-ETS group with a pass rate of $67 \%$ (4 of 6 ). All additional analysis looked at only these 2 groups. Pass rates were statistically discernibly higher for those completing the ETS curriculum compared with the non-ETS group $(P=.01)$ (Fig $2, B)$. 


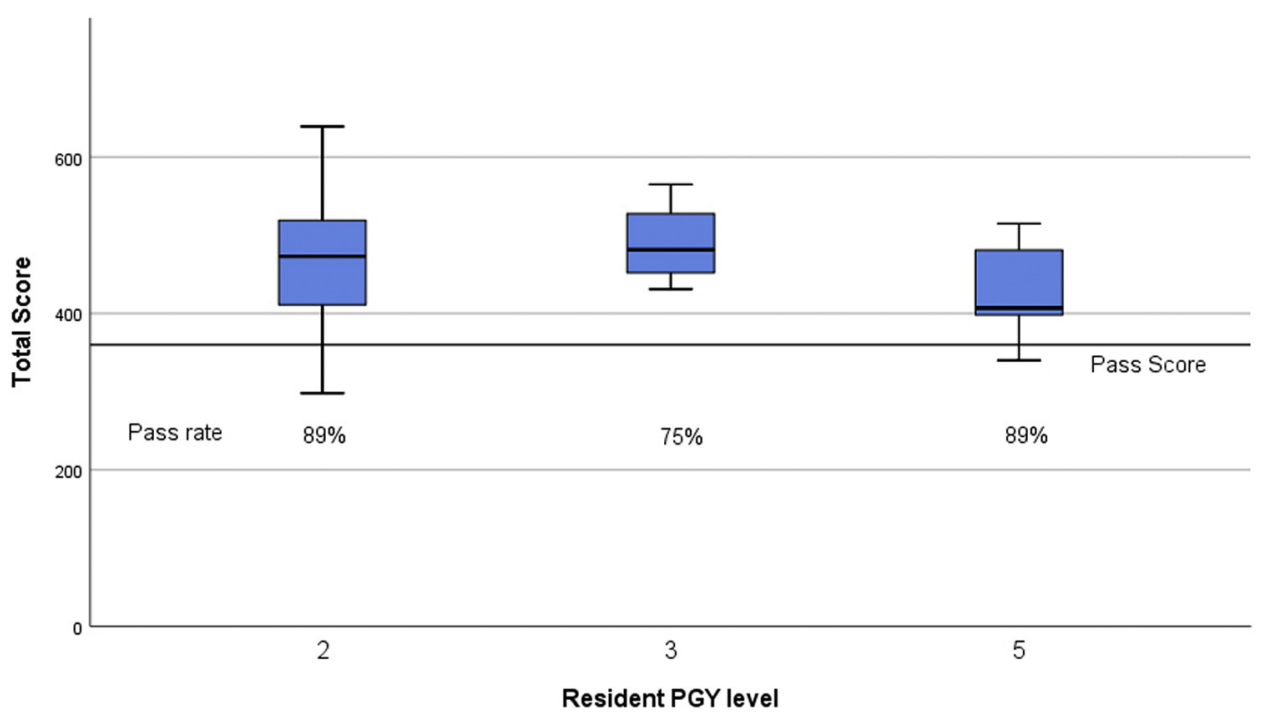

Fig 3. FES exam total score by PGY level. FES, Fundamentals of Endoscopic Surgery.

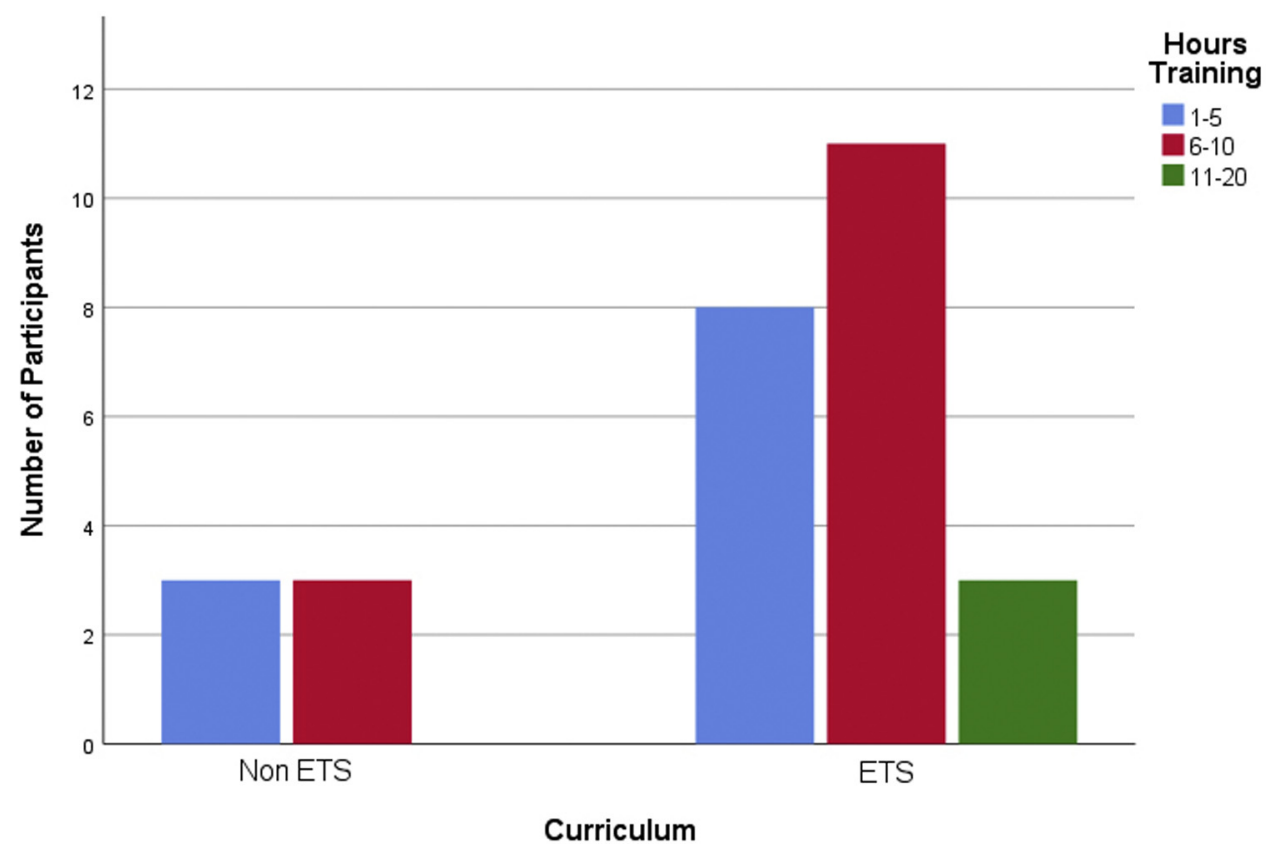

Fig 4. Time spent training for FES skills exam. FES, Fundamentals of Endoscopic Surgery.

FES pass rates and total scores were not discernibly different across PGYs (Fig $3 ; P>.1$ ). For residents completing the ETS curriculum, total FES scores were discernibly higher than those who did not complete the ETS curriculum $(472 \pm 69$ vs $389 \pm 41, P<.01)$. Three out of 5 task scores were also discernibly higher for residents who completed the ETS curriculum: Nav1 $80 \pm 12$ vs $67 \pm 10, P=.02$; Loop $36 \pm 27$ vs $8 \pm 13, P=.02$; and Retro3 $89 \pm 10$ vs $71 \pm 7, P=$ .02 . Scores for mucosal inspection and tool targeting were not discernibly different: $79 \pm 16$ vs $64 \pm 17, P=.053$; and $89 \pm 7$ vs $96 \pm$ $9, P=.074$.

Self-reported simulation training time for residents completing the ETS curriculum was reasonable, with $92 \%$ spending 10 hours or less on task training. Figure 4 shows the distributions of training time required to complete the ETS curriculum.

\section{Discussion}

The ABS recommends completing the FEC by the PGY4 year, but currently no data exists regarding the optimal timing of endoscopy training and FES testing. Furthermore, there have been conflicting data regarding the optimal time to introduce either simulated or clinical endoscopic training into residency program curricula to optimize preparation for FES testing. Our study provides insight into these questions and also examines the amount of time required for training on task-specific endoscopic skills.

The national first-time pass rate on the FES test is $81 \%{ }^{12}$ The majority of data published regarding simulation curriculum development and preparation for the FES examination have been produced by a small number of collaborating groups. Additionally, 
many residency programs do not likely employ these published curricula and use internally unique programs that may or may not be grounded in solid learning theory. As a result, it is impossible to fully analyze trends for how institutions nationwide meet the FEC requirements and prepare for the FES examination. Based on available data and how the FEC was developed, it is likely that training based solely on clinical endoscopy experience, including using a competency measurement tool such as the Global Assessment of Gastrointestinal Endoscopic Skills ${ }^{13}$ or the Assessment of Competency in Endoscopy ${ }^{14}$ is sufficient to prepare residents to pass the FES skills exam. This method would shift FES testing to higher PGY levels to allow time to gain sufficient clinical endoscopy experience. However, previous studies have shown that reliance on clinical exposure that meets only minimum ABS requirements results in prohibitive FES skills test fail rates ranging from $25 \%$ to $40 \% .{ }^{6}$ Failure later in residency can result in difficulties for senior residents requiring remediation to meet requirements to sit for the ABS qualifying examination.

Our group has previously published FES skills testing results after the implementation of a SBML curriculum using the ETS physical training simulator. This study showed that novice endoscopists, PGY1 and PGY2, can pass the FES skills examination when training to a mastery standard. ${ }^{9}$ Our current study shows the effect of implementing a SBML curriculum across an entire residency program, particularly early in residency at the PGY2 level.

The PGY2 residents trained as part of the ES rotation were endoscopically inexperienced, with some receiving their first experience with an endoscope during the rotation. After participation in ES rotation, no PGY2 resident attained the ABS-mandated minimum required number of endoscopies before FES testing. Despite this inexperience, the pass rate for ETS participants far exceeded the national average, and all ETS-trained residents achieved total FES skills test scores that compare favorably with scores from endoscopists who have performed $>300$ clinical endoscopic procedures. $^{9}$

When looking at individual task scores, the ETS curriculum led to discernibly higher scores on 3 tasks when compared with the non-ETS group. In particular, this included Loop2 reduction, which has been shown to cause difficulty for testers in other publications. ${ }^{7}$ Despite scoring higher, 6 (19\%) residents still failed to reduce even 1 loop on the final FES skills test. Although this is fewer than the 3 (50\%) residents for the non-ETS group, better simulation training for successful loop reduction on the FES skills exam is still in need. It is hard to know how often during performance of clinical endoscopy that residents are allowed to perform loop reduction independently as time, patient safety, and comfort considerations may lead faculty endoscopists to perform this part of the procedure more frequently.

The amount of time required to complete a SBML curriculum may initially deter program directors from implementing it as the backbone for skills training. Mastery learning breaks the mold of time or repetition guided curricula and has no specifications of adequate or inadequate training time. This approach allows learners to progress through different educational units at their own pace to achieve mastery of each task. Here, it permits timely completion of training and protects against undertraining due to overconfidence in skill. Even with implementation of mastery learning, nearly all residents using the ETS curriculum completed simulation training in 10 hours or less. Given that post-training performance is similar to endoscopists with $>300$ cases of clinical experience, this represents a great return on the investment for time spent training.

These results reinforce the strength of early implementation of FES skills training and testing using a SBML curriculum. Additionally, repeated results achieving exceedingly high first-time pass rates over a period of 5 academic years by largely novice endoscopists show the consistency of applied mastery learning theory and that completion of FES testing can be successfully carried out early in residency.

Despite the positive results of the ETS curriculum for junior residents, there are limitations to the data presented here. First, although data has been collected over 5 academic years, the overall sample size is still relatively small. Second, our results represent data obtained from our institution and may not be uniformly generalized to all general surgery residency programs. We are fortunate to have a robust simulation center equipped with multiple ETS simulator models as well as the GI Mentor II. We are also a SAGES FES test center, which allows our residents to comfortably test and train in the same learning environment. Currently, the ETS system is available for $<\$ 10,000$, and a functional endoscopy tower can often be repurposed from a retired clinical unit or acquired online for $\sim \$ 20,000 .^{15}$ Although these costs are cheaper than virtual reality trainers, ${ }^{16}$ it may be financially unfeasible for some general surgery residency programs to purchase this training equipment. Additionally, each year, we typically have 1 or 2 Surgical Education and Simulation Fellows who have previously completed the curriculum during their ES rotation and passed the FES exam available to orient trainees to the simulator, coach them through the curriculum, and track progress of residents as they approach the test.

Third, as part of a grant from SAGES, all residents were pretested on the FES skills examination before beginning the curriculum. The impact of the ability to take the FES skills examination as a pretest on total test scores before training remains unclear. The interval between pre- and post-training testing was typically between 2 and 4 weeks, which should allow for "washout" of skills acquired from the original assessment. The 2019-2020 academic year is the first in which our PGY2 residents in the ES rotation will have no experience with the FES skills examination exercises before certification testing. FES test scores at a collaborating institution decreased the year after discontinuation of pretesting, and this was identified as one possible cause. ${ }^{7}$ In the 2019-2020 academic year so far, using the ETS, we have trained and tested an additional 5 PGY2 residents and 1 PGY5 resident without FES skills pretesting and have had no failures on the FES examination to date.

The FEC is designed to ensure that surgeons possess the technical skills required to manage common gastrointestinal diseases with flexible endoscopy. We have developed and broadly implemented a SBML curriculum using the Endoscopy Training System because of its ability to develop the skills required for surgical endoscopists as measured by the FES skills test. There are currently no published studies showing the effect of SBML training with the ETS or any other platform on other outcomes in clinical flexible endoscopy. This will be the focus of future work.

In conclusion, through the use of a SBML curriculum for flexible endoscopy, novice endoscopists can gain the knowledge and skills required to pass the FES skills test, typically with fewer than 10 hours of cumulative endoscopic simulation training. Scores achieved by this group are similar to those with a high level of clinical endoscopic experience that is seldom met by graduating general surgery chief residents. For program directors who are looking for the optimal timing to schedule an endoscopy rotation to meet FEC requirements while ensuring high performance on the FES exam, these goals can be reliably accomplished as early as the PGY2 level.

\section{Funding/Support}

No outside support was received for this research. 


\section{Conflict of Interest/Disclosure}

Christopher Dyke, MD; Brenton R. Franklin, MD, MHPE, FACS; and W. Brian Sweeney, MD, FACS have no potential conflicts of interest to disclose. E. Matthew Ritter, MD, MHPE, FACS received royalties and grant support from the Henry M. Jackson Foundation for the Advancement of Military Medicine.

\section{Acknowledgments}

The views expressed in this presentation are those of the authors and do not reflect the official policy of the Department of Army/Navy/Air Force, Department of Defense, or the U.S. government.

\section{References}

1. American Board of Surgery. Flexible endoscopy curriculum for general surgery residents. https://www.absurgery.org/xfer/abs-fec.pdf. Accessed February 27, 2020.

2. Placek SB, Franklin BR, Ritter EM. Simulation for surgical endoscopy. In: The Comprehensive Textbook of Healthcare Simulation: Simulation for Surgery and Surgical Subspecialties Edition. Switzerland: Springer International Publishing; 2019:221-231.

3. Hashimoto DA, Petrusa E, Phitayakorn R, Valle C, Casey B, Gee D. A proficiencybased virtual reality endoscopy curriculum improves performance on the fundamentals of endoscopic surgery examination. Surg Endosc. 2018;32: 1397-1404.

4. Guzzetta AA, Weis JJ, Hennessy SA, et al. Proficiency-based preparation significantly improves FES certification performance. Surg Endosc. 2018;32: 4451-4457.
5. Franklin BR, Placek SB, Gardner AK, et al. Preparing for the American Board of Surgery Flexible Endoscopy Curriculum: development of multi-institutional proficiency-based training standards and pilot testing of a simulation-based mastery learning curriculum for the Endoscopy Training System. Am J Surg. 2018;216:167-173.

6. Gardner AK, Scott DJ, Willis RE, Van Sickle K, Truitt MS, Uecker J, et al. Is current surgery resident and GI fellow training adequate to pass FES? Surg Endosc. 2017;31:352-358.

7. Weis JJ, Scott DJ, Busato L, Hennessy SA. FES exam outcomes in year two of a proficiency-based endoscopic skills curriculum. Surg Endosc. 2020;34:961-966.

8. Texas Association of Surgical Skills Laboratories. TASSL Curricula: Flexible Endoscopy Curriculum. Accessed February 21, 2020.

9. Ritter EM, Taylor ZA, Wolf KR, et al. Simulation-based mastery learning for endoscopy using the endoscopy training system: a strategy to improve endoscopic skills and prepare for the fundamentals of endoscopic surgery (FES) manual skills exam. Surg Endosc. 2018;32:413-420.

10. Ritter EM, Scott DJ. Design of a proficiency-based skills training curriculum for the fundamentals of laparoscopic surgery. Surg Innov. 2007;14: 107-112.

11. Scott DJ, Ritter EM, Tesfay ST, Pimentel EA, Nagji A, Fried GM. Certification pass rate of $100 \%$ for fundamentals of laparoscopic surgery skills after proficiencybased training. Surg Endosc. 2008;22:1887-1893.

12. Lineberry M, Matthew Ritter E. Psychometric properties of the Fundamentals of Endoscopic Surgery (FES) skills examination. Surg Endosc. 2017;31: 5219-5227.

13. Vassiliou MC, Kaneva PA, Poulose BK, et al. Global Assessment of Gastrointestinal Endoscopic Skills (GAGES): a valid measurement tool for technical skills in flexible endoscopy. Surg Endosc. 2010;24:1834-1841.

14. ASGE Training Committee, Sedlack RE, Coyle WJ, et al. ASGE's assessment of competency in endoscopy evaluation tools for colonoscopy and EGD. Gastrointest Endosc. 2014;79:1-7.

15. Limbs and Things Ltd. Endoscopy Training System. https://limbsandthings. com/us/products/kkmw39/kkmw39-endoscopy-training-system/. Accessed February 21, 2020.

16. Triantafyllou K, Lazaridis LD, Dimitriadis GD. Virtual reality simulators for gastrointestinal endoscopy training. World J Gastrointest Endosc. 2014;6: 6-12.

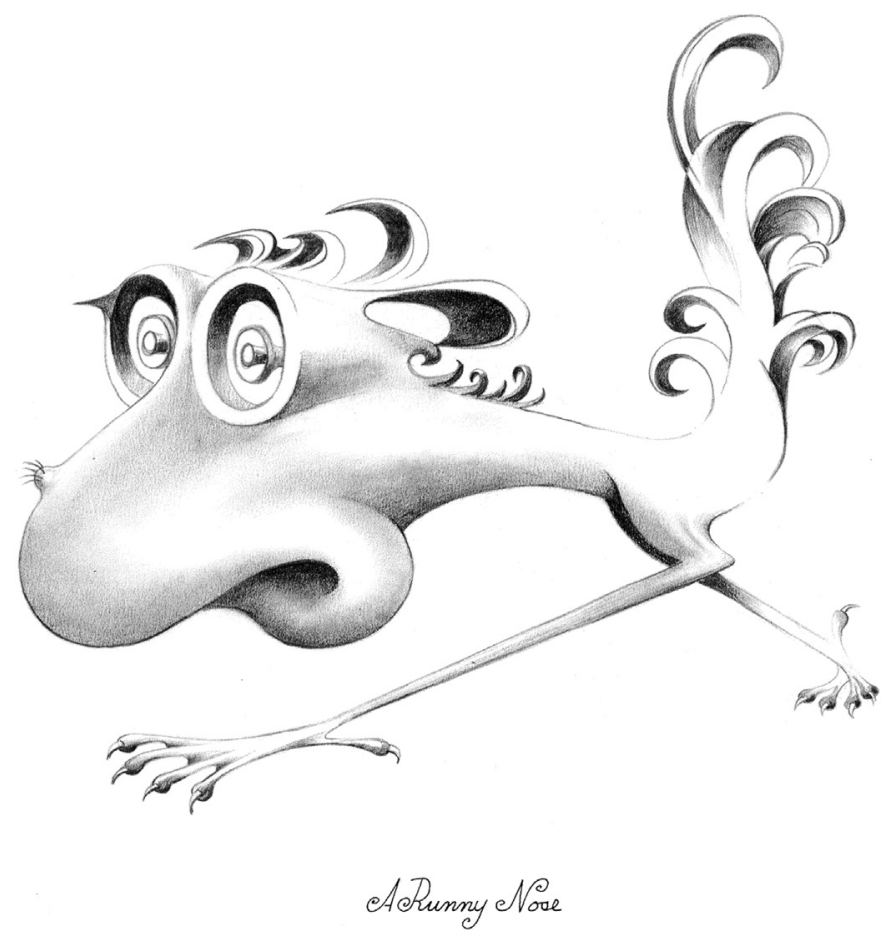

